\title{
A quality-controlled global runoff data set
}

\section{Arising from: N. Gedney et al. Nature 439, 835-838 (2006)}

Gedney et $a l^{1}$ attribute an increase in the twentieth-century continental runoff to the suppression of plant transpiration by $\mathrm{CO}_{2}$ induced stomatal closure, by replicating a continental runoff data set ${ }^{2}$. However, we have concerns about this data set and the methods used to construct it, in addition to those already raised ${ }^{3}$, which we believe may undermine their conclusions.

The continental runoff data set covers the period 1875-1994 and the start and end dates of runoffobservations are listed ${ }^{2}$ for each of 221 constituent stations. A wavelet-based runoff 'reconstruction' methodology ${ }^{2}$ is used to infill any missing data and extend records forwards (to 1994) and backwards (to 1875) where necessary. In addition to previous concern ${ }^{3}$ about the use of runoff stations subject to confounding anthropogenic influences, such as reservoirs, and aspects of the runoff reconstruction methodology, it seems that the runoff reconstruction methodology is based on the best correlation against one of ten unspecified reference stations ${ }^{2}$. There is no discussion about whether these reference stations are spatially and temporally representative of the full range of runoff regimes being reconstructed around the world and not subject to confounding anthropogenic influences. The sensitivity of the final reconstructed runoff to the choice of these reference stations is not properly addressed ${ }^{2,4}$.

A fundamental requirement of a runoff data set used in an attribution study, such as that by Gedney et $a L^{1}{ }^{1}$, is that it is representative of the observed runoff conditions around the world. The concerns outlined above and previously ${ }^{3}$ about the reconstruction methodology call into question the degree to which the runoffdata set used $^{2}$ is representative of those conditions. Key to this is a discussion of the percentage of continental runoff that is reconstructed (infilled or extrapolated), which is not presented ${ }^{12,4}$. Based on the start and end dates of the runoff records ${ }^{2}$, extrapolation from 10-20 years of observed runoff data to the centennial scale 4 has been applied to at least $34 \%$ of the stations. This is likely to be an underestimate, because not all stations have complete records between these dates.

Gedney et $a L^{1}$ analyse continental runoff records based on observations from at least $20 \%$ of the total river basin area, which could be taken to mean that up to $80 \%$ of the continental runoff is reconstructed, not observed, for some periods of their analysis. Without knowing the degree to which the runoff data set is reconstructed and therefore representative, conclusions based on replication of that runoff data in a modelling (attribution, for example) analysis are speculative.

Considering the serious concerns about the continental runoff data set underlying the results of Gedney et $a L^{1}$, their conclusion that an increase in twentieth-century continental runoff is attributable to the suppression of plant transpiration by $\mathrm{CO}_{2}$-induced stomatal closure is called into question. This highlights the need for the development of a quality-controlled, freely available, global runoff data set, which can be used with confidence (or at least informed caution) in future studies.

Murray C. Peel, Thomas A. McMahon

Department of Civil \& Environmental

Engineering, University of Melbourne,

Victoria, 3010, Australia

e-mait: mpeel@unimelb.edu.au

1. Gedney, N. et al. Nature 439,835-838 (2006)

2. Labat, D, Goddéris Y, Yrobst, IL.\& Guyot, J.L. Adv. Water Resour. 27, 631-642 (2004)

3 Legates, D.R, Lins, H.F.\& McCabe, G. I.Adv. Water Resau. 28,1310-1315 (2005)

4. Labat, D, Goddéris Y, Probst, LL.\& Guyot, J.L. Adv. Water Resaur. 28, 1316-1319 (2005).

dol:10.1038/hature 05480

\section{CONTINENTAL RUNOFF}

\section{Gedney et al. reply}

\section{Replying to: M. C. Peel \& T. A. McMahon Nature 444, doi: 10.1038/nature05480 (2006)}

Peel and McMahon ${ }^{1}$ argue that limitations of the continental runoff data set ${ }^{2}$ we use in our study ${ }^{3}$ call our main conclusion into question - that is, that changes in twentieth-century continental-scale runoff are partly attributable to the suppression of $\mathrm{CO}_{2}$-induced stomatal closure. We support Peel and McMahon's pleas for better and more complete runoff data, but we remain confident in our conclusions, as explained here.

First, we do not rely on the conclusion of Labat et $a l^{2}$ that there is a "link between global warming and the intensification of the global hydrological cycle" being correct, which has been questioned 4 . As we are accounting for anthropogenic influences in our study ${ }^{3}$, their presence in basins used in the runoff data set is also not an issue here.

Second, our statistical analysis includes data uncertainty. We use a restricted maximumlikelihood technique that takes account of temporal correlation in the uncertainty, as we expect the data-set errors to be time-dependent. This produces wider and more realistic error bars than standard fitting techniques. As we are modelling timescales from annual to multi-decadal, our ability to reproduce the runoff data set at such a wide spread of temporal scales acts as a strong crosscheck. Yet, we still find that the effects of $\mathrm{CO}_{2}$-induced stomatal closure are detectable at a statistically significant level.

Third, we find that, over every continent except Europe, induding the direct $\mathrm{CO}_{2}$ effect produces temporal behaviour that is closer to the observational data than when only the climate effect is included. This is also the case over Europe if we incorporate the net effect of human water consumption ${ }^{5,6}$. Of the nonclimatic mechanisms considered, only the direct $\mathrm{CO}_{2}$ effect has both the temporal and spatial structure that is consistent with the observational runoff ${ }^{2}$. The likelihood that such a signature is in the observational data set by chance is therefore small, given that any errors in the data set ${ }^{2}$ are likely to vary spatially and temporally.

For the reasons given ${ }^{3}$, we consider periods when the observed runoff areal coverage is at least $20 \%$ of the total, thereby avoiding some of the interpolation and gap-filling problems referred to by Peel and McMahon ${ }^{1}$. In addition, as already mentioned ${ }^{3}$ and as discussed in more detail here, our results are consistent when we consider different time periods. The proportion of areal observation coverage in the runoff data set at a given year can be calculated from tables in ref. 2 . However, the total observed discharge is likely to be more relevant for assessing the influence of reconstructed data ${ }^{2}$. Indeed, we can obtain a good estimate of the proportion of observed runoff within the data set $^{2}$ by considering readily available measurements (Global Runoff Data Centre, Koblenz, Germany) from those rivers with large annual discharges (more than $6,000 \mathrm{~m}^{3} \mathrm{~s}^{-1}$ ). The total runoff of this subset accounts for about $80 \%$ of gauged runoff total in the data set ${ }^{2}$, making it a reasonable representation. In this sub-sample, $63-92 \%$ of the observations are available over the $1950-95$ period.

As already mentioned ${ }^{3}$, our results are actu- 